\title{
Soins palliatifs - ou «Mourir est un art»
}

\section{Jean Martin}

Dr méd., membre de la rédaction

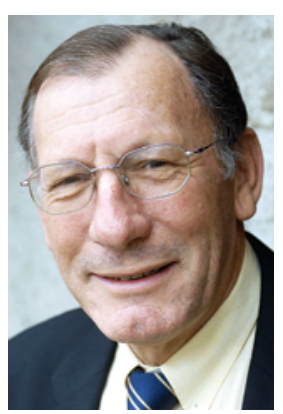

L'association Curaviva avait son Congrès «Personnes âgées» 2017 (le septième) à Montreux les 19 et 20 septembre. Gros succès d'audience avec 1300 participants. A noter que c'était en même temps la «Global Ageing Conference» du Global Ageing Network, réunissant des professionnels intéressés de plusieurs dizaines de pays. On y a entendu des conférences plénières d'orateurs connus de divers domaines comme l'ancienne conseillère fédérale Micheline Calmy-Rey, le cuisinier de renommée mondiale Anton Mosimann, le charismatique professeur de médecine génétique Markus Hengstschläger, de Vienne.

Une session parallèle m’a particulièrement intéressé, intitulée «Sterben ist (k)eine Kunst» (mourir est/n'est pas un art). Les responsables de deux institutions y ont présenté leurs pratiques en matière de soins palliatifs et de fin de vie. D'une part le KZU (Kompetenzzentrum Pflege und Gesundheit) de Bassersdorf (ZH), d'autre part l'établissement Erlenhaus, de Engelberg (OW). J'aimerais évoquer ici des éléments apportés par la directrice de la Erlenhaus, Theres Meierhofer, qui est aussi

\section{C'est le patient lui-même qui devrait être le chef d'orchestre!}

membre du Groupe de travail Palliative Care de son canton. Son institution héberge 50 résidents et doit assumer le décès, chaque année, de $40 \%$ d'entre eux environ (cette proportion est semblable au KZU susmentionné). Un sérieux défi, à l'évidence. Elle a abordé quatre thèmes: le travail interdisciplinaire, l'accompagnement des proches, la question des rituels, le soulagement des symptômes - en faisant référence à plusieurs reprises aux Recommandations de Bigorio émanant de Palliative.ch.

Dans son propos: Notre action est basée sur la relation, en tenant compte des ressources et intérêts de chacun et de la vie en commun, conformément à notre devise «En sécurité pendant la vie - en sécurité au moment de mourir». L'équipe de la Erlenhaus s'efforce par exemple de trouver, avec les patients, l'occasion de revisiter leur histoire et de parler de ce qu'ils vivent dans la maison.
Ces échanges montrent, aux côtés d'autres moyens bien sûr, un effet bénéfique sur les symptômes.

Elle a souligné l'importance des rituels. Il n'est pas question (comme cela a pu être le cas dans le passé) de traiter une mort au sein de l'EMS de manière aussi discrète que possible voire à la sauvette. «On sort par la grande porte», dit-elle, décrivant un cérémonial particulier mis en œuvre quand le cercueil quitte la maison, en présence du personnel. Une réunion a lieu avec les résidents, pour parler du disparu et de ce que son départ suscite chez chacun(e).

Beaucoup d'attention est portée aux proches; on leur offre même le petit-déjeuner au lendemain du décès on est dans une communauté rurale, avec sa vie locale et ses caractéristiques, où tout le monde se connaît. Th. Meierhofer a noté que cela permettait à l'équipe de moduler/adapter les contacts avec les proches, en fonction par exemple de difficultés éventuelles connues au sein de telle famille (c'est évidemment plus malaisé à réaliser en milieu urbain). Après les différentes phases du processus, il y a une rencontre de l'équipe où on discute cette question: tout au long du séjour de ce résident et jusqu'à la fin, «avons-nous fait juste?».

La formation - de base et continue - en soins palliatifs de tous les acteurs est fondamentale, inspirée par la formule "Accompagner dans la vie et dans la fin de vie est notre activité centrale» (Kerngeschäft - core business). Formation où il s'agit de débattre d'attention à l'autre, d'appréciation et de prise en compte des valeurs (les siennes et celles des autres), d'attitudes professionnelles et personnelles. La dimension spirituelle y tient une place. Des journées de formation ont été organisées pour le Conseil de fondation du home (où a été traitée aussi la question de l'assistance au suicide). Enfin, à propos de travail interdisciplinaire: ce qui se passe autour et avec un patient ressemble à la vie d'un orchestre, avec de multiples intervenants, chacun jouant sa partition. A cet égard, l'oratrice dit que, dans la mesure du possible, c'ést le patient lui-même qui devrait être le chef d'orchestre - pour ce qui le concerne, les professionnels ayant pour but de lui permettre de l'être! 